\title{
Concomitant Autoimmune Diseases in Patients With Sarcoidosis in Turkey
}

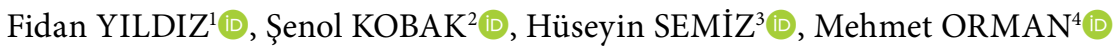 \\ ${ }^{1}$ Department of Chest Disease, Okan University Faculty of Medicine, İstanbul, Turkey \\ ${ }^{2}$ Department of Internal Medicine, Division of Rheumatology, Istinye University Faculty of Medicine, İstanbul, Turkey \\ ${ }^{3}$ Department of Internal Medicine, Ege University Faculty of Medicine, İzmir, Turkey \\ ${ }^{4}$ Department of Biostatistics and Medical Informatics, Ege University Faculty of Medicine, İzmir, Turkey
}

\begin{abstract}
Objectives: This study aims to determine the frequency and characteristics of autoimmune diseases associated with sarcoidosis patients. Patients and methods: The study included 131 sarcoidosis patients ( 36 males, 95 females; mean age 46.1 years; range, 20 to 82 years). Demographic, clinical, laboratory and radiological data of patients were evaluated retrospectively. The characteristics of autoimmune diseases associated with sarcoidosis (sarcoidosis-overlap group) patients and isolated sarcoidosis (isolated sarcoidosis group) were analyzed and compared.

Results: Concomitant autoimmune diseases were detected in 15 (11.5\%) (5 males, 10 females; mean age 50.8 years; range, 26 to 58 years) of the 131 patients with sarcoidosis and their mean disease duration was three months (range, 1 to 30 months). When compared with isolated sarcoidosis patients, more hand finger joint involvement, rheumatoid factor (RF) positivity, higher erythrocyte sedimentation rate (ESR) and less nonsteroidal anti-inflammatory drugs (NSAIDs) usage were found in the sarcoidosis-overlap group $(p=0.035, p=0.049, p=0.015, p=0.018$, respectively). There were no statistically significant differences between the two groups when evaluated for demographic, clinical parameters and disease-modifying antirheumatic drugs usage.

Conclusion: Concomitant autoimmune diseases in patients with sarcoidosis may be rarely seen. These patients are characterized with more hand finger joint involvement, RF positivity, higher ESR and less NSAIDs usage. Multicenter, prospective studies involving large numbers of patients are needed to understand whether the association of sarcoidosis-autoimmune diseases is based only on coincidence or on a common etiopathogenesis. Keywords: Autoimmune diseases, concomitant, sarcoidosis.
\end{abstract}

Sarcoidosis is a systemic inflammatory disease of unknown etiology that is characterized by the presence of non-caseating granulomas in affected tissues. ${ }^{1}$ The immunopathogenesis of sarcoidosis is complex and not completely understood. It is postulated that exposure to an unknown environmental antigen in a predisposed individual leads to an abnormal type I immune response. Sarcoidosis may present with different clinical findings such as lung, skin and eyes involvement. It may mimic some manifestations of a number of autoimmune diseases and/or develop concomitantly with them. ${ }^{2}$ Sarcoidosis may coexist with different rheumatic autoimmune diseases. Some authors have suggested that concurrent disease is rare and may be due to a nonspecific granulomatous reaction and is not truly sarcoidosis. There are also studies reporting that sarcoidosis may aggravate or mimic the clinical and laboratory findings of these diseases. ${ }^{3}$ Sarcoidosis does not meet the criteria for autoimmune disease. However, many

Received: May 30, 2019 Accepted: August 29, 2019 Published online: January 08, 2020

Correspondence: Fidan Yıldız, MD. Okan Üniversitesi Tıp Fakültesi Göğüs Hastalıkları Anabilim Dalı, 34959 Tuzla, İstanbul, Türkiye. Tel: +90 532 - 5614777 e-mail: fidanyildiz@yahoo.com 
case reports have suggested that there is an association between various autoimmune diseases and sarcoidosis. ${ }^{4}$ To our knowledge, although no data exist of such an association yet, given their prevalence, it is extremely unlikely for patients to develop both diseases concomitantly.

In this study, we aimed to determine the frequency and characteristics of autoimmune diseases associated with sarcoidosis patients.

\section{PATIENTS AND METHODS}

One hundred and thirty-one sarcoidosis patients (36 males, 95 females; mean age 46.1 years; range, 20 to 82 years) followed up by Şifa University Rheumatology Clinic between January 2012 and May 2016 were retrospectively evaluated. Patients' data were collected and documented using our computer system. Biochemical, serological, laboratory tests and imaging techniques were used for diagnosis. Biopsy specimens taken from different tissues (lymph nodes, skin, parotid gland, penis) were used for histological verification of sarcoidosis, and diagnosis was confirmed as the pathologists showed the non-caseating granulomas. The diagnosis of sarcoidosis was established according to histopathologic, clinical, radiologic, and laboratory investigations. Other possible causes of granulomatous diseases (tuberculosis, bacterial, fungal infections) were excluded. Laboratory investigations were performed on all patients, and their routine biochemistry, acute phase reactants, serum angiotensin converting enzyme, calcium and hydroxy-vitamin D3 levels were checked. Plain chest radiography and thorax computed tomography (CT) were performed for staging of sarcoidosis. Diagnosis of concomitant autoimmune disease was established according to the diagnostic criteria of the involved diseases. Clinical, demographical, laboratory, radiological and histological data of both groups (sarcoidosisoverlap and isolated sarcoidosis groups) of patients were compiled and analyzed. The study protocol was approved by the Şifa University Hospital Ethics Committee. A written informed consent was obtained from each patient. The study was conducted in accordance with the principles of the Declaration of Helsinki.

\section{Statistical analysis}

Data were analyzed by the IBM SPSS version 20.0 software (IBM Corp., Armonk, NY, USA). Cross tables were used in analysis of data and Chi-square and Fisher's exact tests were performed where appropriate. The data were given as frequency and percentage. Statistical significance threshold was considered as 0.05 .

\begin{tabular}{|lccc|}
\hline Table 1. Clinical characteristics of sarcoidosis-overlap cohort $(\mathrm{n}=15)$ \\
\hline & $\mathrm{n}$ & $\%$ & Mean \\
\hline Sex & & & \\
Male & 5 & 33.3 & \\
Female & 10 & 66.6 & \\
Mean age (year) & & & 50.8 \\
Mean disease duration (month) & & & \\
Race & 3 & & \\
Caucasian & & & \\
Concomitant autoimmune disease & & & \\
Ankylosing spondylitis & & & \\
Rheumatoid arthritis & & & \\
Scleroderma & 4 & 26.6 \\
Sjögren's syndrome & 3 & 20 \\
Familial Mediterranean fever & 1 & 1 \\
Still's disease & 1 & 6.6 \\
Gouty arthritis & 1 & 6.6 \\
Graves' disease & 1 & 6.6 \\
Hashimoto thyroiditis & 1 & 6.6 \\
Immune thrombocytopenic purpura & 1 & 6.6 \\
& 1 & 6.6 \\
& 1 & 6.6 \\
\hline
\end{tabular}




\section{RESULTS}

We identified 15 patients (11.5\%) (5 males, 10 females; mean age 50.8 years; range, 26 to 58 years) with concomitant autoimmune disease and sarcoidosis and their mean disease duration was three months (range, 1 to 30 months). The most frequent concomitant autoimmune diseases were ankylosing spondylitis (AS) and rheumatoid arthritis (RA), whereas other patients were diagnosed as Sjögren's syndrome, scleroderma, gouty arthritis, immune thrombocytopenic purpura, Hashimoto thyroiditis and Graves' disease, respectively. Two patients had autoinflammatory diseases (Still's disease and familial Mediterranean fever [FMF]) (Table 1). Sarcoidosis presented prior to the autoimmune disease in four of 15 patients (26.7\%). An autoimmune disease presented prior to sarcoidosis in 9 of 15 patients (60\%). Both diseases presented concomitantly in two patients (13.3\%). An evaluation of our cohort of 131 sarcoidosis patients for system and organ involvement showed erythema nodosum in 59 patients (45\%), uveitis in 18 patients (13.7\%), and bone involvement, lupus pernio myositis and neurosarcoidosis in one patient (0.76\%). Plain chest radiography and thorax CT results showed stage 0 (extrapulmonary) disease in five patients (3.8\%), stage 1 (bilateral hilar lymphadenopathy) in 64 patients $(48.8 \%$ ), stage 2 (bilateral hilar lymphadenopathy + pulmonary infiltrate) in 45 patients (34.3\%), stage 3 (only pulmonary

Table 2. Comparison between sarcoidosis-overlap and isolated sarcoidosis patients according to demographic, clinical and laboratory features $(n=131)$

\begin{tabular}{|c|c|c|c|c|c|c|c|}
\hline \multirow[b]{2}{*}{ Features } & \multicolumn{3}{|c|}{ Sarcoidosis-overlap group $(\mathrm{n}=15)$} & \multicolumn{3}{|c|}{ Isolated sarcoidosis group $(\mathrm{n}=116)$} & \multirow[b]{2}{*}{$p$} \\
\hline & $\mathrm{n}$ & $\%$ & Mean & $\mathrm{n}$ & $\%$ & Mean & \\
\hline Age (year) & & & 50.8 & & & 48.5 & 0.588 \\
\hline $\begin{array}{l}\text { Sex } \\
\quad \text { Female }\end{array}$ & 10 & 66.7 & & 86 & 89.6 & & 0.538 \\
\hline Disease duration (month) & & & 3 & & & 12 & 0.409 \\
\hline Arthralgia & 15 & 100 & & 102 & 87.9 & & 0.368 \\
\hline Fatigue & 8 & 53.3 & & 69 & 59.5 & & 0.649 \\
\hline Arthritis & 11 & 73.3 & & 66 & 56.9 & & 0.224 \\
\hline Hand joints involvement & 2 & 13.3 & & 1 & 0.9 & & 0.035 \\
\hline Neurosarcoidosis & 1 & 6.7 & & 0 & 0 & & 0.115 \\
\hline Erythema nodosum & 6 & 40 & & 53 & 45.7 & & 0.677 \\
\hline Lupus pernio & 0 & 0 & & 1 & 0.9 & & 1.000 \\
\hline Löfgren syndrome & 2 & 13.3 & & 17 & 14.7 & & 1.000 \\
\hline Myopathy & 0 & 0 & & 1 & 0.9 & & 1.000 \\
\hline Uveitis & 4 & 26.7 & & 14 & 12.1 & & 0.128 \\
\hline Bone involvement & 0 & 0 & & 1 & 0.9 & & 1.000 \\
\hline Elevated serum ACE & 8 & 53.3 & & 51 & 44 & & 0.493 \\
\hline Elevated serum ESR & 12 & 80 & & 54 & 46.6 & & 0.015 \\
\hline Elevated serum CRP & 7 & 46.7 & & 51 & 44 & & 0.843 \\
\hline RF positivity & 4 & 26.7 & & 11 & 9.5 & & 0.041 \\
\hline CS use & 7 & 46.7 & & 37 & 31.9 & & 0.254 \\
\hline NSAIDs use & 3 & 20 & & 61 & 52.6 & & 0. 018 \\
\hline HQ use & 3 & 20 & & 12 & 10.3 & & 0.379 \\
\hline MTX use & 1 & 6.7 & & 2 & 1.7 & & 0.308 \\
\hline AZA use & 1 & 6.7 & & 0 & 0 & & 0.115 \\
\hline $\mathrm{CD}$ use & 1 & 6.7 & & 3 & 2.6 & & 0.389 \\
\hline
\end{tabular}


infiltrate without hilar lymphadenopathy) in seven patients $(5.3 \%)$, and stage 4 (pulmonary fibrosis) in 10 patients (7.6\%) with sarcoidosis. Histopathological verification of sarcoidosis was performed by endobronchial ultrasound and mediastinoscopy and also by demonstrating noncaseating granulomas through biopsies of the skin, axillary lymphadenopathy, penile mass and parotid gland. Biopsy was not taken in patients presenting with typical clinical features of Löfgren syndrome (fever, bilateral hilar lymphadenopathy, erythema nodosum, ankle arthralgia/arthritis). All 15 sarcoidosis-overlap patients had biopsyproven diagnosis. Table 2 shows the clinical features of sarcoidosis-overlap and isolated sarcoidosis groups. When compared with isolated sarcoidosis patients, more hand finger joint involvement, rheumatoid factor (RF) positivity, higher erythrocyte sedimentation rate and less nonsteroidal anti-inflammatory drugs usage were found in sarcoidosis-overlap group $(p=0.035$, $p=0.049, \quad p=0.015, \quad p=0.018$, respectively). Arthritis, uveitis and neurosarcoidosis were more common in sarcoidosis-overlap group, but this was not statistically significant. There were no statistically significant differences between both groups when evaluated for demographic, clinical parameters and disease-modifying antirheumatic drugs usage.

\section{DISCUSSION}

Our study showed close association between sarcoidosis and autoimmune diseases. The most frequent concomitant autoimmune diseases were AS and RA. To our knowledge, there are various case reports and only two studies in the literature showing the association of sarcoidosis and AS. A hospital-based study showed higher prevalence of radiological sacroiliitis in patients with sarcoidosis (6.6\%) compared with the prevalence of spondyloarthritis (1.9\%) in the normal population. ${ }^{5}$ These results and the possible association were further supported by our previous study where sacroiliitis was noticed in $14.3 \%$ of sarcoidosis patients in Turkey. ${ }^{6}$ However, these studies have still not answered the main question of whether there is a true association or incidental coexistence between sarcoidosis and AS. Probably, both diseases share common pathogenic mechanisms, particularly in terms of some pro-inflammatory cytokines. This hypothesis was partly supported by successful treatment of AS and pulmonary sarcoidosis by infliximab. Sarcoidosis may mimic or coexist with RA. ${ }^{7}$ Furthermore, RF and antinuclear antibody positivity were reported in patients with sarcoidosis. ${ }^{8}$ In a study, the rate of anti-cyclic citrullinated peptide antibodies was determined as $4.7 \%$ in patients with sarcoidosis. ${ }^{9}$ These antibodies may be important in the differential diagnosis of sarcoidosis-RA overlap syndrome and RA. In our study, we found three sarcoidosis patients with concomitant RA, and this was in accordance with previous cases reported in the literature. ${ }^{7}$ Numerous case reports are available on coexistence of sarcoidosis and primary Sjögren's syndrome. ${ }^{10}$ Sicca symptoms and parotid gland enlargement may also be present in patients with sarcoidosis. Exocrine gland biopsy, serologic tests, and clinical findings should be taken into consideration in order to differentiate both diseases. Positive serologic tests (anti-Sjögren's syndrome A [anti-Ro] and antiSjögren's syndrome B [anti-La] antibodies) support Sjögren's syndrome while the presence of uveitis supports sarcoidosis. Clinical features of both diseases are very similar. Judson et al. ${ }^{11}$ reported concomitant sarcoidosis and connective tissue diseases (CTD) in 15 patients followed-up at two medical centers. The patients were predominantly females and scleroderma was the most common CTD. As we report in this study, coexistence of sarcoidosis with gouty arthritis, Still's disease and FMF is rather rare. The relationship between gouty arthritis and sarcoidosis was reported years ago. ${ }^{12}$ Because hyperuricemia and similar clinical pictures may occur in both diseases, demonstration of monosodium urate crystals phagocytozed by neutrophils is diagnostic for gouty arthritis. Autoimmune thyroid diseases are the most frequently reported autoimmune comorbidities associated with sarcoidosis. In a casecontrol study including 111 cases of sarcoidosis, a higher prevalence of clinical hypothyroidism (5.3\%) and Graves' disease (4.0\%) was reported in female patients with sarcoidosis compared with age- and sex-matched controls. ${ }^{13}$ In addition, a higher prevalence of Hashimoto thyroiditis (11\%) in pulmonary sarcoidosis patients, and significant association between thyrotoxicosis and sarcoidosis were reported. ${ }^{14,15}$ In our study, we detected two patients with thyroid autoimmune diseases coexisting with sarcoidosis. 
Our study has some limitations. The restricted data from only one center and relatively small number of patients would not allow us to generalize our results. Moreover, since the clinical presentations of sarcoidosis may vary with respect to ethnicity, the results of the current study might not be generalizable to other populations.

In conclusion, our study showed the association of sarcoidosis with some autoimmune diseases in Turkey. The study demonstrated that sarcoidosis patients were more likely associated with AS, RA and autoimmune thyroid disease. We advise physicians to be alert against autoimmune comorbidities in sarcoidosis patients to ensure early diagnosis and treatment. More prospective studies are needed in this regard.

\section{Declaration of conflicting interests}

The authors declared no conflicts of interest with respect to the authorship and/or publication of this article.

\section{Funding}

The authors received no financial support for the research and/or authorship of this article.

\section{REFERENCES}

1. Newman LS, Rose CS, Maier LA. Sarcoidosis. N Engl J Med 1997;336:1224-34.

2. Baughman RP, Lower EE. Features of sarcoidosis associated with chronic disease. Sarcoidosis Vasc Diffuse Lung Dis 2015;31:275-81.

3. Chatham W. Rheumatic manifestations of systemic disease: sarcoidosis. Curr Opin Rheumatol 2010;22:85-90.

4. Wu CH, Chung PI, Wu CY, Chen YT, Chiu YW, Chang YT, et al. Comorbid autoimmune diseases in patients with sarcoidosis: A nationwide case-control study in Taiwan. J Dermatol 2017;44:423-430.

5. Erb N, Cushley MJ, Kassimos DG, Shave RM, Kitas GD. An assessment of back pain and the prevalence of sacroiliitis in sarcoidosis. Chest 2005;127:192-6.

6. Kobak S, Sever F, Ince O, Orman M. The prevalence of sacroiliitis and spondyloarthritis in patients with sarcoidosis. Int J Rheumatol 2014;2014:289454.

7. Fallahi S, Collins RD, Miller RK, Halla JT. Coexistence of rheumatoid arthritis and sarcoidosis: difficulties encountered in the differential diagnosis of common manifestations. J Rheumatol 1984;11:526-9.

8. Kobak S, Yilmaz H, Sever F, Duran A, Sen N, Karaarslan A. The prevalence of antinuclear antibodies in patients with sarcoidosis. Autoimmune Dis 2014;2014:351852.

9. Kobak S, Ylmaz H, Sever F, Duran A, Sen N. Anticyclic citrullinated peptide antibodies in patients with sarcoidosis. Sarcoidosis Vasc Diffuse Lung Dis 2014;31:206-10.

10. Hansen SR, Hetta AK, Omdal R. Primary Sjögren's syndrome and sarcoidosis: coexistence more than by chance? Scand J Rheumatol 2008;37:485-6.

11. Judson MA, Shapiro L, Freitas S, Polychronopoulos VS, Highland KB. Concomitant sarcoidosis and a connective tissue disease: review of the clinical findings and postulations concerning their association. Respir Med 2013;107:1453-9.

12. Kaplan H, Klatskin G. Sarcoidosis, psoriasis, and gout: syndrome or coincidence? Yale $\mathrm{J}$ Biol Med 1960;32:335-52.

13. Antonelli A, Fazzi P, Fallahi P, Ferrari SM, Ferrannini E. Prevalence of hypothyroidism and Graves disease in sarcoidosis. Chest 2006;130:526-32.

14. Nakamura H, Genma R, Mikami T, Kitahara A, Natsume $\mathrm{H}$, Andoh $\mathrm{S}$, et al. High incidence of positive autoantibodies against thyroid peroxidase and thyroglobulin in patients with sarcoidosis. Clin Endocrinol (Oxf) 1997;46:467-72.

15. Rajoriya N, Wotton CJ, Yeates DG, Travis SP, Goldacre MJ. Immune-mediated and chronic inflammatory disease in people with sarcoidosis: disease associations in a large UK database. Postgrad Med J 2009;85:233-7. 